\title{
INTELIGENCIA EMOCIONAL: LAS EMOCIONES SON ÚTILES
}

\section{EMOTIONAL INTELLIGENCE: EMOTIONS ARE USEFUL}

María García Gómez1: Universidad Católica San Antonio de Murcia. España. mggomez@ucam.edu

\section{RESUMEN}

Las emociones quedaron en un segundo plano con el triunfo de la razón durante siglos. Fueron concebidas como algo primitivo e instintivo que obstaculizaba el flujo de la razón. Sin embrago, estamos asistiendo al resurgir del proceso emocional como algo útil y necesario gracias a la difusión de conceptos como la inteligencia emocional. La funcionalidad de las emociones como elemento necesario para la supervivencia de las especies destaca su valor adaptativo. Las investigaciones que exploran sus bases neuroanatómicas demuestran la relevancia de la información que proporcionan las emociones en el proceso de toma de decisiones y su función determinante en nuestro comportamiento diario. Las emociones son funcionales a nivel intrapersonal, interpersonal, movilizan la acción y comunican. Dada la relevancia de las emociones como fuente de conocimiento, es esencial aprender a gestionarlas para poder adaptarnos al medio y mejorar nuestro bienestar y calidad de vida.

PALABRAS CLAVE: razón, emoción, inteligencia, cognición, adaptación, funcionalidad, neuroanatomía.

\section{ABSTRACT}

Over centuries, emotions were not considered useful due to the triumph of reason as the main knowledge. Emotions were viewed as primitive and instinctive responses, which are not associated with cognitive functions. However, we are witnessing emotion resurgence. Most people consider emotion as a useful and necessary process thanks to concepts such as emotional intelligence. Functionality of emotions as a key element for survival is related to its adaptive value. In the body of research, we explore their neuroanatomical, bases and pointed out the high relevance of their role in the decision-making process and daily behavior. Emotions are functional as interpersonal, intrapersonal level, and mobilize action and communicate information. Due to the relevance of emotions as a source of knowledge, it is essential to learn how to manage them in order to adapt to the environment and improve our wellbeing and quality of life.

KEY WORDS: reason, emotion, intelligence, cognition, adaptation, functionality, neuroanatomy.

\footnotetext{
1 María García Gómez: Profesora colaboradora en la Universidad Católica de San Antonio. Departamento de Ciencias Sociales Jurídicas y de la Empresa.
} 


\section{Cómo citar el artículo:}

García Gómez, M. (2014). Inteligencia emocional: las emociones son útiles. Revista de Ciencias de la Comunicación e Información, 19, 23-36.

http://doi.org/10.35742/revistacccomunicacioneinformacion.2014.19.23-36

\section{INTRODUCCIÓN}

Desde finales de los años noventa estamos asistiendo a nueva conceptualización de las emociones que algunos denominan incluso revolución, la cual ha venido desarrollándose en diversos ámbitos, fundamentalmente ha afectado a la psicología, la educación y la sociedad.

Bisquerra (2002) asegura que esta difusión de las emociones viene marcada por el incremento de las publicaciones científicas, el desarrollo de las neurociencias y la enorme difusión de obras como la de Goleman (1995).

La inteligencia emocional (IE) implica aprender a administrar las emociones para que éstas trabajen a favor de la persona (Garaigordobil y Oñederra, 2010), además describe una serie de aptitudes que son complementarias a la inteligencia académica (Bernarás, Garaigordobil y de las Cuevas, 2011).

La validez de este constructo así como su utilidad queda patentada por los estudios que demuestran su valor como predictor de resultados significativos para la vida. Diversos investigadores han incrementado su validez divergente al relacionarla con aspectos fundamentales de la psicología y psiquiatría (Lizeretti y Extremera, 2011).

Una gran cantidad de investigaciones sustentan la premisa de que la inteligencia emocional es un predictor de la salud y el bienestar (véase Bastian, Burns \& Nettelbeck, 2005; Brackett \& Mayer, 2003; Brackett, Mayer, \& Warner, 2004; Ciarrochi, Chan, \& Caputi, 2000; Martins, Ramalho, \& Morin, 2010; Trinidad \& Johnson, 2002), rendimiento académico (e.g. Di Fabio \& Palazzeschi, 2009; MacCann et al., 2011; Qualter, Gardner,Hutchinson, Pope \& Whiteley, 2012) y está relacionada con variables relevantes en el ámbito laboral (Kerr et al., 2006; Kidwell, Hardesty, Murtha, \& Sheng, 2011; Lopes et al., 2006; Mueller \& Curhan, 2006; O'Boyle et al., 2010; Rosete \& Ciarrochi, 2005; Vitello-Cicciu, 2003).

Un estudio de metaanálisis desarrollado por Schutte, Malouff, Thorsteinsson, Bhullar y Rooke (2007) encuentra que los estudios sustentan la relación entre IE con salud mental, física y psicosomática.

Respecto a su validez predictiva, estudios de metaanálisis como el de Van Rooy \& Viswesvaran (2004) apoyan su validez. Según estos autores, las medidas de IE muestran una validez operacional de $.10, .24$ y .24 respectivamente para aspectos académicos, vitales y laborales.

La inteligencia emocional también ha sido asociada con signos de salud mental como menores niveles de depresión, ansiedad y estrés (Slaski \& Cartwright, 2002; 
Tsaousis \& Nikolaou, 2005), y características positivas como comportamiento prosocial, empatía, mejores relaciones familiares y de amistad, buena calidad de interacciones sociales, salud general y satisfacción en el trabajo (Lopes et al., 2004).

Aquellas personas que son capaces de expresar y entender las emociones, comprender el significado de las experiencias emocionales y regular sus sentimientos, presentan mayor ajuste psicológico y social (Mayer, Roberts y Brasade, 2008).

Además, se considera que la inteligencia emocional es un mediador entre la satisfacción con la vida y el estrés. Ciarrochi, Deane y Anderson (2002) encuentran que la IE modera la relación entre los estresores vitales y la salud mental, resultados que son replicados por Bhullar, Schutte y Malouff (2012) posteriormente. El mayor nivel de IE previene el desarrollo de estados emocionales desadaptativos como la ansiedad y la depresión (Bhullar, Schutte y Malouff, 2012). Las investigaciones muestran que aquellos sujetos con mayor nivel de inteligencia emocional tienen mayor nivel de estados de ánimo positivos, además de reparar mejor los estados de ánimo negativos (Schutte et al. 2002).

Si tal y como afirman Bastian et al. (2005) la relevancia de un constructo psicológico depende de su incidencia sobre aspectos significativos de la vida, con los datos expuestos provenientes de las investigaciones desarrolladas para su estudio, queda justificada su notable importancia y el estudio empírico del mismo.

La inteligencia emocional ha demostrado su importancia y con ello ha contribuido al cambio en la visión de las emociones como un proceso útil y necesario para la supervivencia y adaptación al medio.

\section{OBJETIVOS}

En esta disertación se pretende promover el interés por las emociones como una fuente de conocimiento útil y exponer el proceso emocional como complemento del proceso cognitivo y no como procesos opuestos o excluyentes.

\section{METODOLOGÍA}

Este artículo es de corte ensayístico. La base metodológica es fundamentalmente teórica. Se ha redactado mediante una síntesis de los principales estudios actuales tras la revisión bibliográfica y hemerográfica de las principales fuentes documentales. Los estudios que se han consultado relacionan las emociones y la cognición como una unión indivisible y necesaria, destacando el valor y función adaptativa de las emociones.

\section{RESULTADOS}

La definición de las emociones no ha sido un tema exento de controversia. Delimitar un concepto poco visible e intangible no es tarea fácil. Existen múltiples definiciones de lo que es emoción y dependiendo del paradigma imperante en cada época podemos hallar diferentes conceptualizaciones. 
De las definiciones de emoción existentes se presenta la siguiente por considerarla más específica, clara y concreta.

Fernández-Abascal, Jiménez y Martín (2003) la definen como:

Un proceso que implica una serie de condiciones desencadenantes (estímulos relevantes), la existencia de experiencias subjetivas 0 sentimientos (interpretación subjetiva), diversos niveles de procesamiento cognitivo (procesos valorativos), cambios fisiológicos (activación), patrones expresivos y de comunicación (expresión emocional), que tiene unos efectos motivadores (movilización para la acción) y una finalidad: que es la adaptación a un entorno en continuo cambio. (Fernández-Abascal y Jiménez, 2003, p. 40).

\subsection{Funcionalidad de las emociones}

Scherer (1996) destaca que la principal función de las emociones es detectar y preparar los sistemas de respuesta, de este modo, según aumenta la intensidad emocional también lo hace la velocidad de respuesta a los estímulos ambientales, favoreciendo la supervivencia de la especie.

Sus principales funciones son descritas por Martínez-Sánchez, Páez y Ramos (2005), los cuales destacan las siguientes funcionalidades:

1. Funciones intrapersonales. Sirven de coordinador de los sistemas de respuesta subjetivo, fisiológico y conductual, lo cual nos lleva a realizar conductas como lucha o retirada que pueden salvarnos en situaciones determinadas. También son ayuda a la hora de tomar decisiones, favoreciendo el razonamiento en muchas ocasiones, algo que no se había considerado tradicionalmente.

2. Funciones interpersonales. Mediante la expresión facial y corporal de las emociones podemos comunicar a los demás cómo nos sentimos, lo cual permite a los demás reaccionar conforme a nuestras emociones, su beneficio depende de las circunstancias, es decir, que esto no significa que siempre sea bueno expresar nuestras emociones, sabemos que en situaciones, por ejemplo, laborales, debemos ocultar muchas veces nuestras emociones, en otras ocasiones sí nos ayuda expresarlas a relacionarnos adecuadamente con los demás así como sentirnos mejor. Numerosos estudios señalan que la represión de las emociones tiene efectos perjudiciales para nuestra salud mental y física.

3. Las emociones y la motivación. Ambos términos se encuentran estrechamente relacionados desde el origen latino del concepto: "movere", las emociones movilizan a la acción a través de la activación fisiológica. También motivan la conducta de los otros, por ejemplo, el llanto hace que los demás traten de ayudarnos y consolarnos.

En nuestra sociedad se ha reprimido habitualmente la tristeza, siendo concebida como signo de debilidad, sin embargo, se sabe de su carácter adaptativo ya que nos permite conservar recursos mediante la retirada, para más tarde volver con energías renovadas a tratar de afrontar de nuevo aquello que no logramos anteriormente. En 
otras ocasiones puede no ser adaptativa la acción que promueve la emoción, por ejemplo el miedo en el caso de las fobias produce conductas de evitación que pueden llegar a limitar ampliamente la vida cotidiana de una persona. También cabe diferenciar los efectos a corto y largo plazo, pues lo que puede ser beneficioso en un plazo puede no serlo en otro.

4. Las emociones comunican. Para Schward y Clore (1983) la función principal de las emociones es dar a conocer a los demás información personal. Pero además supone una fuente de información interna que podemos usar a la hora de tomar decisiones. Por tanto, comunican a los demás y también a nosotros mismos.

5. Las emociones son un índice de señalamiento de la información. Permiten que valoremos un hecho como placentero o displancentero dependiendo que cómo nos sintamos ante él, es decir, nos dicen si un hecho nos gusta o nos disgusta. Nos señalan, en este sentido, la relevancia de determinados estímulos.

Por tanto, los autores señalan que a pesar de su carácter adaptativo, las emociones pueden no ser funcionales y ocasionar en el individuo trastornos como la ansiedad o la depresión.

En cuanto a su funcionalidad debe ser tenido en cuenta:

- La experiencia emocional siempre es adaptativa pero la expresión de la emoción puede no serlo (e.g., Keltner y Gross, 1999).

- La conducta emocional puede ser funcional a corto plazo pero puede no serlo a largo plazo (e.g., Greenberg y Pavio, 2000).

- Las emociones pueden ser disfuncionales en función de su intensidad, frecuencia, duración y aparición inapropiada (e.g., Cano-Vindel y MiguelTobal, 2001).

Siguiendo a estos autores, el proceso emocional sigue las siguientes fases: evaluación de una situación antecedente, cambios fisiológicos, expresión motora, efectos motivadores con tendencia a la acción, sentimientos subjetivos y afrontamiento.

El desencadenante de la emoción puede ser un estímulo externo, una situación determinada, o un estímulo interno, un pensamiento, después el organismo realiza una valoración del mismo, se activa fisiológicamente, expresa corporalmente la emoción y posteriormente actúa. Además los desencadenantes de la emoción pueden clasificarse como intrapersonales, así encontramos respuestas selectivas propias de nuestra especie a determinados estímulos, por ejemplo, los estímulos novedosos nos elicitan mayores respuestas emocionales y observamos miedos comunes que protegen a la especie. Por otro lado, la historia personal del sujeto, todo lo que le acontece desde que se forma, va creando huellas emocionales que producirán respuestas inconscientes que le harán responder en milisegundos a situaciones determinadas, sin que la persona sea consciente de su origen.

Respecto a la expresión y el control de las emociones, la cultura juega un papel determinante. El modo de gestionar las emociones es aprendido dentro de un 
determinado contexto. La familia en la que crecemos también moldea nuestras experiencias emocionales así como las capacidades emocionales que desarrollemos (Martínez-Sánchez, Páez y Ramos, 2005).

Para la Ecología Emocional las emociones cumplen con la función de guía de nuestro mapa mental, son una brújula, debemos atenderlas, escucharlas, tenerlas en cuenta para tomar decisiones y sabes gestionarlas de un modo adaptativo y sostenible. En este contexto, son patrimonio intangible de la humanidad, sin ellas no podríamos sobrevivir (Conangla y Soler, 2011).

\subsection{Emociones y cognición}

Tradicionalmente el saber era un asunto que competía a los filósofos. Mediante la reflexión y la razón nos proporcionaban sus teorías sobre la esencia, las propiedades, causas y efectos de las cosas.

La primera teoría de las emociones fue desarrollada por Platón, cuya trilogía consistía en dividir la mente o el alma en razón, apetito y espíritu, que se corresponde en la actualidad con emoción, cognición y motivación.

Para Aristóteles las dos dimensiones del alma, la racional y la irracional, forman una unidad, y es considerado precursor de las teorías cognitivas de la emoción al considerar que la emoción tiene rasgos cognitivos como las expectativas y las creencias.

Según estos filósofos las emociones no tienen significado ni función, son opuestas al hombre evolucionado racional, los animales son instintos, emociones, y el hombre es un ser perfecto racional que no debe dejarse influenciar por las emociones que lo perturban. De este modo postulan la existencia de cuatro tipos de emociones: el anhelo de los bienes futuros, la alegría de los bienes presentes, el temor a males futuros y la aflicción por los males presentes. En este contexto, las emociones son consideradas enfermedades crónicas que amenazan a la razón (Casado y Colmo, 2006).

El dualismo cartesiano divide el alma y el cuerpo en dos sustancias distintas, y establece la existencia de seis emociones básicas a partir de las cuales se construye el resto: el asombro, el amor, la alegría, la tristeza, el odio y el deseo. Esta distinción de las emociones básicas perdura con modificaciones hasta la actualidad, y el pensamiento de Descartes ha tenido una influencia enorme en la concepción de las emociones del mundo occidental.

A pesar de concebirla como algo que perturba la razón del hombre, los pensadores han reconocido la fuerza de las emociones como propulsor del individuo. Para Kant algunas de las emociones ayudan a la existencia, por lo que cobra tintes más positivos.

Los grandes pensadores han influido hasta nuestros días, contribuyendo a la visión de las emociones como propias de animales más primitivos que el hombre y otorgando un sesgo negativo a todo comportamiento que esté basado en fuentes emocionales. 
Aunque en la actualidad las teorías no niegan la funcionalidad y significado de las emociones, se considera que las emociones dependen de la razón.

Para la perspectiva cognitiva ambas, emoción y cognición, son consideradas igualmente importantes, pero la emoción es fruto de las interpretaciones que hacemos de nuestro entorno. Por tanto, esta perspectiva no supone una ruptura completa con la visión tradicional de las emociones. Autores como Zajonc (1980) plantean que antes de la interpretación se pueden dar reacciones afectivas inconscientes que influyen en el comportamiento fuera del alcance de la cognición.

El modo en el que han sido desterradas las emociones a lo largo de las épocas, queda recogido por Zaccagnini (2004). Este autor realiza una revisión histórica sobre el modo en el que se han tomado las emociones a lo largo de la historia. Zaccagnini (2004) resalta el hecho de que las emociones siempre han existido y no es algo nuevo pensar que condicionan el comportamiento humano. Lo que sí sufre cambios es la importancia que les ha dado a lo largo de la historia.

Siguiendo la exposición de Zaccagnini (2004) encontramos que en la Grecia Clásica se pensaba que las emociones no eran una condición inherente al ser humano, sino que eran impuestas por los Dioses del Olimpo a los hombres para controlarlos. Por tanto, el hombre no tenía control sobre ellas, eran gobernados por ellas sin poder hacer nada para impedirlo. Se trataba del modo que los Dioses tenían de gobernar al hombre, y por tanto éste debía resignarse a las mismas pasivamente, de ahí el nombre de "pasiones". Tras este modelo fatalista grecolatino, surge otro propio de la religión judeocristiana. Las emociones son internas al hombre, no impuestas de modo externo, y son causadas por las tentaciones del mundo externo. El ser humano debe controlarlas, luchar contra ellas para que no le aparten del buen camino. En este contexto tornan en algo pecaminoso, algo que debe ser reprimido, y por tanto, son negativas. En el Renacimiento la razón pasa a ocupar un papel central en la cultura llamada "La Ilustración" que dará comienzo a la época de la "modernidad". Este modelo se consolida en la Revolución Francesa y se mantiene hasta la actualidad.

La vida social e individual está gobernada por la "razón humana", el ser humano sigue dividido en cuerpo (emociones) y alma (razón), tal y como propugnaba el dualismo cartesiano. En la modernidad la inteligencia racional es lo "serio", lo correcto, el camino de la ciencia, la tecnología, el avance. Las emociones deben ser controladas ya que son irracionales y no ayudan al trabajo y a los aspectos serios de la vida, por lo que su expresión se debe supeditar a los momentos lúdicos y de ocio, para que no obstaculicen nuestro mundo racional. Tras la Segunda Guerra Mundial comienza el periodo que llamamos "posmodernidad", donde empieza a cuestionarse la supremacía y papel único de la razón. Se cuestiona la división propuesta por el dualismo, la razón y la emoción no son opuestas, incluso se complementan en el comportamiento psicológicamente bien adaptado. Las emociones dejan de ser impulsos ciegos que controlan al hombre y que hay que evitar, forman parte de las relaciones interpersonales, las decisiones que tomamos diariamente, no son cálculos "fríos", las emociones orientan y guían nuestras vidas. 
Lo que se ha puesto de moda es entonces la interpretación de las emociones como una fuerza positiva que nos proporciona información y nos guía para mejorar nuestras vidas y desarrollar un comportamiento adaptativo. Como dijo Pascal, "el corazón tiene razones que la razón no conoce".

Comienza una nueva necesidad de conocer y entender las emociones en lugar de reprimirlas. La razón no pierde importancia, sino que su papel ahora es instrumental para la consecución de fines, pero necesita de la emoción para elegir bien esos fines y mantener la conducta necesaria para su consecución. Estamos asistiendo al "renacimiento" de las emociones. Parece ser un tema de moda desde finales del siglo XX. No es que sea algo nuevo que las emociones condicionan nuestro comportamiento cotidiano, pero sí lo es el papel que les asignamos en nuestra cultura. Prueba de ello es el aumento en los últimos años de webs dedicadas a la emoción, pasando de unas 20 mil en el año 1995 a unas 900 mil en el año 2003. Nuestra sociedad "posmoderna" caracterizada por el individualismo precisa de una dimensión psicológica que incluya el conocimiento y manejo de las emociones dado el inmenso poder que tienen las emociones en el mismo. Las emociones pueden facilitarnos nuestra vida diaria, siendo una fuerza propulsora que nos ayuda a sentirnos dichosos, mientras en otras ocasiones origina conflictos emocionales con nosotros mismos y con lo demás. Los estados emocionales tienen una influencia permanente y son claves de felicidad, bienestar psicológico y calidad de vida (Zaccagnini, 2004).

Otra gran influencia en las concepciones actuales de la emoción es la teoría de Darwin. En 1872 publica "La expresión de las emociones en el hombre y los animales", en ella expone tres principios que continúan vigentes en las teorías actuales: "hábitos útiles asociados" según el cual las emociones poseen funciones adaptativas; "antítesis" que se refiere a los diferentes modos de expresión de las diversas emociones; "acción directa al sistema nervioso" que se refiere a la asociación de los principios anteriores así como la actividad fisiológica que subyace en las emociones. Para Darwin las emociones tienen una función adaptativa para la supervivencia, y gracias a sus estudios se produjo un incremento del interés por este ámbito. De sus investigaciones se derivan algunos supuestos básicos: la expresión emocional es universal, está determinada genéticamente y cumple una función comunicativa ante situaciones de emergencia que incrementa la posibilidad de supervivencia (Fernández-Abascal et al., 2010).

\subsection{Bases neuroanatómicas de las emociones}

La investigación sistemática de las emociones viene marcada por el filósofo y psicólogo americano William James, cuyo trabajo inicial What is an emotion publicado en 1884, defiende la hipótesis de que la respuesta fisiológica era la causa de la respuesta emocional, es decir, estamos tristes porque lloramos y tenemos miedo porque corremos, por tanto, la sensación fisiológica precede a la experiencia emocional. Las emociones serían respuestas cognitivas provocadas por la estimulación periférica. Esta teoría junto con toda una tradición de la cual James fue predecesor, es llamada teorías periféricas de las emociones, que fueron criticadas por conceder poca importancia a los procesos evaluativos y por las evidencias 
posteriores que constatan la existencia de sustratos cerebrales específicos de las emociones (Le Doux, 2000).

En 1927 Walter Cannon y Phillip Bard presentan una teoría contraria a las periféricas, según la cual el control de las emociones se relaciona con estructuras talámicas y describen circuitos neurales implicados en la expresión y experiencia emocional. Tras esta teoría se suceden las investigaciones en torno a la base cerebral de las emociones. En 1930 tras numerosas evidencias se considera el lóbulo límbico como principal implicado en el control emocional. Unos años más tarde, en 1937 es James Papez a quien se debe el famoso circuito de Papez el cual vincula las emociones con el hipotálamo, el núcleo talámico anterior y la corteza cingulada. La corteza controlaría las emociones y el hipotálamo la expresión de las mismas (Le Doux, 2000).

En 1952, MacLean introduce el término sistema límbico, en el cual añade al propuesto por Papez, otras estructuras cerebrales que se encuentran también relacionadas con las emociones y son el núcleo amigdalino, el septum y la corteza prefrontal. Estas teorías continúan otorgando una funcionalidad biologicista y, por tanto, adaptativa de las emociones, las cuales aseguran la supervivencia.

Aunque estas teorías contribuyeron al aumento de evidencia sobre las bases neuroanatómicas de la emoción, supusieron un apoyo para la escisión de la cognición y la emoción, ya que se suponía que las emociones formaban parte de las áreas más subcorticales y primitivas del cerebro, mientras que los procesos cognitivos se encontraban situados en áreas más desarrolladas y filogenéticamente más evolucionadas. Sin embargo, posteriormente se han ido descubriendo otras muchas regiones cerebrales implicadas en los procesos emocionales. La función de control de respuestas de defensa y establecimiento del condicionamiento del miedo ha sido adjudicada en gran medida al núcleo amigdalino. Los núcleos amigdalinos tienen relación con estructuras tan relevantes como la neocorteza, la formación hipocampal y los núcleos talámicos (reciben información del ambiente externo) e hipotalámicos (ambiente interno). Su lugar estratégico le permite por tanto implicarse tanto en la evaluación como en la expresión de emociones (Le Doux, 2000).

El papel fundamental de la amígdala en los procesos evaluativos y, en concreto, en el miedo condicionado, fue claramente descrito por Joseph LeDoux. Según sus investigaciones, la amígdala nos permite primero actuar y después pensar, con las importantes repercusiones que ello tiene para la supervivencia. Otros interesados en este campo como Adolphs y su equipo han constatado que las lesiones bilaterales de la amígdala produce una dificultad para identificar expresiones faciales de miedo, así como problemas para procesar la información amenazante, de modo que se veían envueltas en situaciones que ponían en riesgo su vida (Puigcerver, 2005).

La amígdala posee un rol fundamental en el control y aprendizaje de respuestas de miedo y ansiedad, preservando las situaciones amenazantes para en otro momento futuro responder ante situaciones semejantes. Su modo de funcionar se supone más implícito, mientras el papel del hipocampo es más explícito, consciente y verbalizable, ambos se relacionan estrechamente (Fernández-Abascal et al., 2010). De este modo podemos explicar porque respondemos fisiológica y conductualmente 
ante situaciones que conscientemente no comprendemos o no interpretamos como peligrosas.

Otro grupo de estudio con importantes contribuciones en este terreno ha sido el de Adrian Raine y Antonio Damasio, los cuales destacan la importancia de las regiones orbitofrontales en los procesos de toma de decisiones. Además relacionan la lesión de tales áreas con el trastorno antisocial, así como alteraciones de conducta y agresividad. La corteza prefrontal también ha sido ampliamente relacionada con las emociones. Las lesiones en estas áreas en el hemisferio izquierdo producen estados depresivos, por lo que se ha relacionado esta parte del córtex con las emociones positivas.

Del control de las emociones es protagonista la región ventromedial, su descubrimiento se debe al famoso caso de Phineas Gage, cuyo lóbulo frontal se vio atravesado por una barra, las consecuencias fueron un cambio de personalidad radical y permanente.

Por citar una última aportación relevante en el estudio de las bases neuronales de las emociones, destacamos la hipótesis del marcador somático descrita por Antonio Damasio. El autor describe en su obra El error de Descartes en 1994 la relación entre la emoción y la razón, de este modo las experiencias pasadas de recompensa y castigo producen estados corporales que guían al organismo a tomar decisiones. Las situaciones quedan asociadas con respuestas somáticas como cambios vegetativos, musculares, neuroendocrinos o neurofisiológicos, que son señales inconscientes "marcadores somáticos" que en un futuro nos ayudarán de modo inconsciente a la hora de tomar decisiones (Fernández-Abascal et al., 2010). Los sustratos neuroanatómicos que se han asociado a tales marcadores son córtex prefrontal ventromedial y el orfitofrontal. Sus hipótesis se sustentan en varios experimentos, uno de ellos ha sido bastante difundido, se denomina la tarea de apuestas. A grandes rasgos, la tarea trata de que el participante debe elegir entre cuatro montones de cartas, dependiendo de cuál elija va obteniendo ganancias o pérdidas económicas, dos de los montones llevan ganancias más discretas pero pocas pérdidas, por lo que son más ventajosos que los otros en los cuales sucede al contrario. Los individuos, sin ser conscientes de ello, van eligiendo los más ventajosos, eso sí, aquellos que presentan lesiones en el córtex prefrontal ventromedial no pueden valerse de esta información intuitiva inconsciente y fracasan en la tarea al arriesgar demasiado. Estas regiones corticales nos permiten simular mentalmente y de modo inconsciente, las posibles consecuencias de cada una de nuestras acciones para elegir una respuesta adecuada. Al no poder hacer uso de su intuición, de sus marcadores, los participantes lesionados no tomaban las decisiones apropiadas.

La trascendencia de estas aportaciones al tema que nos ocupa es innegable. Siguiendo a Mayer y Salovey (2006), estás investigaciones refutan las ideas de los estoicos cuya visión de las emociones era considerada un obstáculo para la razón. Las emociones pueden ayudar a tomar las decisiones más apropiadas incluso antes que tenga lugar un análisis racional. 


\section{CONCLUSIONES Y DISCUSIÓN}

La inteligencia emocional y el estudio sistemático de las emociones dentro de la ciencia han contribuido a retomar la visión de las emociones como un proceso con una funcionalidad adaptativa.

Mientras que el saber tradicional de los antiguos filósofos concebía la emoción como algo que perturbaba la razón del hombre, a pesar de ello otorgaban poder y fuerza impulsora a las emociones como determinantes del comportamiento.

Investigaciones en el campo de las neurociencias sitúan a las emociones como procesos que bien gestionados contribuyen al buen funcionamiento de la razón y la cognición.

Las emociones nos ayudan en el proceso de toma de decisiones. No son opuestas al proceso cognitivo, sino que lo complementan. Además las emociones son claves en nuestra vida diaria y guían nuestro comportamiento.

La gestión emocional se torna una asignatura básica de la vida si queremos tener calidad de vida y bienestar emocional.

\section{BIBLIOGRAFÍA}

Bastian, V. A., Burns, N. R., \& Nettelbeck, T. (2005). Emotional intelligence predicts lifeskills, but not as well as personality and cognitive abilities. Personality and Individual Differences, 39, 135-145.

Bernarás, E., Garaigordobil, M. y de las Cuevas, C. (2011). Inteligencia emocional y rasgos de personalidad. Boletín de Psicología,103, 75-88.

Brackett, M.A. \& Mayer, J.D. (2003). Convergent, discriminant and incremental validity of competing measures of emotional intelligence. Personality and Social Psychology Bulletin, 29, 1147-1158.

Brackett, M. A., Mayer, J. D., \& Warner, R. M. (2004). Emotional intelligence and its relation to everyday behaviour. Personality and Individual Differences, 36, 1387-1402.

Bastian, V. A., Burns, N. R., \& Nettelbeck, T. (2005). Emotional intelligence predicts lifeskills, but not as well as personality and cognitive abilities. Personality and Individual Differences, 39, 135-145.

Bhullar, N., Schutte, N., \& Malouff, J. M. (2012). Trait Emotional Intelligence as a Moderator of the Relationship Between Psychological Distress and Satisfaction with Life. Individual Differences Research, 1(10), 19-26.

Bisquerra, R. (2002). La competencia emocional. En M. Álvarez y R. Bisquerra, Manual de orientación y tutoría, 144, 69-144. Barcelona: Práxis. 
Cano Vindel, A. \& Miguel Tobal, J. J. (2001). Emociones y salud. Ansiedad y Estrés, 7(2-3), 111-121.

Casado, C. y Colomo, R. (2006). Un breve recorrido por la concepción de las emociones en la filosofía occidental. A parte Rei. Revista de filosofía, 47.

Ciarrochi, J., Chan, A.Y.C. \& Caputi, P. (2000). A critical evaluation of the emotional intelligence construct. Personality and Individual Differences, 28, 539-561.

Ciarrochi, J., Deane, F. P. y Anderson, S. (2002) Emotional intelligence moderates the relationship between stress and mental health. Personality and individual differences, 32, 197-209.

Conangla, M. M. \& Soler, J. (2011). Ecología emocional para el nuevo milenio: el arte de reinventarse a uno mismo. Barcelona: Zenith.

Di Fabio, A., \& Palazzeschi, L. (2009). An in-depth look at scholastic success: Fluid intelligence,personality traits or emotional intelligence? Personality and Individual Differences, 46, 581-585.

Fernández-Abascal, E. G., García, B., Jiménez, M. P., Martín, M. D. y Domínguez, F. J. (2010). Psicología de la Emoción. Madrid: Centro de Estudios Ramón Areces.

Fernández-Abascal, E. G., Jiménez, M. P. y Martín, M. D. (2003). Emoción y Motivación: La adaptación humana I. Madrid: Editorial Centro de Estudios Ramón Areces, S.A.

Goleman, D. (1995). Emotional Intelligence. New York: Bantam.

Garaigordobil, M., y Oñederra, J. A. (2010). Inteligencia emocional en las víctimas de acoso escolar y en los agresores [Emotional intelligence in victims of school bullying and in aggressors]. European Journal of Education and Psychology, 3(2), 243-256.

Greenberg, L. S. \& Pavio, S. (2000). Trabajar con las emociones en psicoterapia. Barcelona: Paidós.

Keltner, D. \& Gross, J. (1999). Functional accounts of emotions. Cognition and Emotion, 5, 467-480.

Kerr, R., Garvin, J., Heaton, N., \& Boyle, E. (2006). Emotional intelligence and leadership effectiveness. Leadership\& Organization Development Journal, 27(4), 265-279.

Kidwell, B., Hardesty, D. M., Murtha, B. R., \& Sheng, S. (2011). Emotional intelligence in marketing exchanges. Journal of Marketing, 75, 78-95. 
Le Doux, J. E. (2000). Emotions circuits in the brain. Annual Review of Neurosciencience, 23, 155-184.

Lizeretti, N. P. y Extremera, N. (2011). Emotional Intelligence and Clinical Symptoms in Outpatients with Generalized Anxiety Disorder (GAD). Psychiatric Quarterly, 82, 253-260.

Lopes, P. N., Brackett, M., Nezlek, J., Schutz, A., Sellin, I., \& Salovey, P. (2004). Emotional intelligence and social interaction. Personality and Social Psychology Bulletin, 30, 1018-1034.

Lopes, P. N., Grewal, D., Kadis, J., Gall, M., \& Salovey, P. (2006). Evidence that emotional intelligence is related to job performance and affect and attitudes at work. Psicothema, 18, 132-138.

Martínez-Sánchez, F., Páez, D. y Ramos Díaz, N. (2005). Emoción y adaptación. Introducción al concepto científico de emoción. En Fernández-Berrocal,P. y Ramos Díaz, N. (Eds): Corazones inteligentes, (pp. 51-75). Barcelona: Kairós.

Martins, A., Ramalho, N., \& Morin, E. (2010). A comprehensive meta-analysis of the relationship between emotional intelligence and health. Personality and Individual Differences, 49, 554-564.

MacCann, C., Fogarty, G. J., Zeidner, M., \& Roberts, R. D. (2011). Coping mediates the relationship between emotional intelligence (EI) and academic achievement. Contemporary Educational Psychology, 36, 60-70.

MacLean, P. D. (1952). Some psychiatric implications of physiological studies on frontotemporal portion of limbic system (visceral brain). Electroencephalography and Clinical Neurophysiology, 4(4), 407-418.

Mayer, J. D., Roberts, R. D., \& Barsade, S. G. (2008). Human abilities: Emotional intelligence. Annual Review of Psychology, 59, 507-536.

Mayer, J. D., \& Salovey, P. (2006). Inteligencia emocional. Mente y Cerebro, 16, 1020.

Mueller, J., \& Curhan, J. (2006). Emotional intelligence and counterpart mood inductionin a negotiation. International Journal of Conflict Management, 17, 110 128.

Puigcerverg ( 2005). Emociones cerebrales. En Fernández-Berrocal, P. y Ramos Díaz, N. (Eds): Corazones inteligentes, (pp. 39-50). Barcelona: Kairós.

Qualter, P., Gardner, K. J., Pope, D., Hutchinson, J. M., \& Whiteley, H. E. (2012). Abilityemotional intelligence, trait emotional intelligence, and academic success in Britishsecondary schools: A 5-year longitudinal study. Learning and Individual Differences, 22, 83-91. 
Rosete, D., \& Ciarrochi, J. (2005). Emotional intelligence and its relationship to workplaceperformance of leadership effectiveness.Leadership Organizational Development Journal, 26, 388-399.

Slaski, M.\& Cartwright, S. (2002). Health performance and emotional intelligence: An exploratory study of retail managers. Stress and Health, 18, 63-68.

Scherer, K.R. (1996). Emotion. En M. Hewstone, W. Stroebe y G.M. Stephenson (Eds.), Introduction to Social Psychology. (pp. 279-315). Oxford: Blackwell.

Schutte, N. S., Malouff, J.M., Simunek, M., McKinley, J. \& Hollander, S. (2002). Characteristic emotional intelligence and emotional well-being. Cognition Emotion, 16(6), 769-785.

Schutte, N. S., Malouff, J. M., Thorsteinsson, E. B., Bhullar, N., \& Rooke, S. E. (2007). A meta-analytic investigation of the relationship between emotional intelligence and health. Personality and Individual Differences, 42, 921-933.

Schwarz, N., \& Clore, G. L. (1983). Mood, misattribution, and judgments of wellbeing: Informative and directive functions of affective states. Journal of personality and social psychology, 45(3), 513.

Trinidad, D. R., \& Johnson, C. A. (2002). The association between emotional intelligence and early adolescent tobacco and alcohol use.Personality and Individual Differences, 32(1), 95-105.

Tsaousis, I. \& Nikolaou, I. (2005). Exploring the relationship of emotional intelligence with physical and psychological health functioning. Stress and Health, 21, 7786.

Van Rooy, D. L., \& Viswesvaran, C. (2004). Emotional intelligence: A meta-analytic investigation of predictive validity and nomological net. Journal of Vocational Behavior, 65(1), 71-95.

Vitello-Cicciu, J. M. (2003). Emotional Intelligence. Nursing Management, 34(10), 1833.

Zaccagnini, J.L. (2004). Inteligencia Emocional. La Relación entre Pensamientos y Sentimientos en la Vida Cotidiana. Madrid: Biblioteca Nueva.

Zajonc, R.B. (1980). Feeling and thinking: Preferences need no inferences. American Psychologist, 35, 151-175. 